\title{
ON THE GENUS OF REDUCIBLE SURFACES AND DEGENERATIONS OF SURFACES
}

\author{
ALBERTO CALABRI, CIRO CILIBERTO, FLAMINIO FLAMINI, RICK MIRANDA
}

\begin{abstract}
In this paper we deal with a reducible projective surface $X$ with so-called Zappatic singularities, which are a generalization of normal crossings. First we compute the $\omega$-genus $p_{\omega}(X)$ of $X$, i.e. the dimension of the vector space of global sections of the dualizing sheaf $\omega_{X}$. Then we prove that, when $X$ is smoothable, i.e. when $X$ is the central fibre of a flat family $\pi: X \rightarrow \Delta$ parametrized by a disc, with smooth general fibre, then the $\omega$-genus of the fibres of $\pi$ is constant.
\end{abstract}

\section{INTRODUCTION}

In this paper we study some topological properties of reducible projective complex surfaces (e.g. unions of planes in a projective space) with so-called Zappatic singularities, which are a generalization of normal crossings. These surfaces naturally occur as central fibres of (embedded) flat degenerations of surfaces, but they are interesting also on their own.

Recall that a projective surface has a Zappatic singular point of type $R_{n}$ [resp. $S_{n}, E_{n}$ ], $n \geqslant 3$, if it is locally analytically isomorphic to the vertex of a cone over a union of lines whose dual graph is a chain of length $n$ [resp. a fork with $n-1$ teeth, a cycle of order $n$ ] and which is projectively normal in the projective space it spans (see Definition 2.1 below).

A Zappatic surface $X$ is a reduced, connected, projective surface which is a union of smooth surfaces and whose singularities are $R_{n^{-}}, S_{n^{-}}, E_{n}$-points, $n \geqslant 3$, and, in codimension one, double curves which are smooth and irreducible along which two irreducible components of $X$ meet transversally (see Definition 2.2). This terminology is justified by interesting papers by Guido Zappa (see e.g. [16, 17]), where degenerations of surfaces to unions of planes with

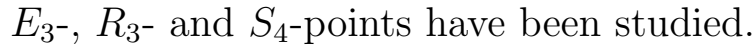

As stick curves are used to study moduli spaces of smooth curves (cf. [8] and [14]), degenerations of surfaces to a Zappatic surface naturally arise in the study of components of moduli spaces of smooth surfaces and their compactifications (see e.g. [3], [4], [6]). We expect that such degenerations will find even more applications, e.g., in the classification of surfaces with low invariants, in braid monodromy computations (see [5], [11], [12], [15]), in the birational classification of higher-dimensional varieties, etc.

In [2], we made a complete analysis of the $K^{2}$ and $\chi$ invariant for Zappatic surfaces and degenerations of smooth surfaces to Zappatic ones. There is one more primary birational invariant for surfaces, namely the geometric genus; this paper is devoted to the analysis of this invariant for Zappatic surfaces and degenerations.

Let us define the $\omega$-genus of a projective variety $Y$ to be

$$
p_{\omega}(Y):=h^{0}\left(Y, \omega_{Y}\right),
$$

where $\omega_{Y}$ is the dualizing sheaf of $Y$. It is just the arithmetic genus, if $Y$ is a reduced curve, and the geometric genus, if $Y$ is a smooth surface.

Mathematics Subject Classification (2000): 14J17; (Secondary) 14B07, 14D06, 14D07, $14 \mathrm{~N} 20$.

The first three authors are member of G.N.S.A.G.A. at I.N.d.A.M. "Francesco Severi". The authors would like to thank L. Badescu and A. Beauville for discussions and references. 
One of the results in this paper is the computation of the $\omega$-genus $p_{\omega}(X)$ of a Zappatic surface $X$ (cf. Theorem 3.1):

Theorem 1. Let $X=\bigcup_{i=1}^{v} X_{i}$ be a Zappatic surface and let $G_{X}$ be its dual graph (cf. Definition 2.4). Consider the natural map $\Phi_{X}: \bigoplus_{i=1}^{v} H^{1}\left(X_{i}, \mathcal{O}_{X_{i}}\right) \rightarrow \bigoplus_{1 \leqslant i<j \leqslant v} H^{1}\left(C_{i j}, \mathcal{O}_{C_{i j}}\right)$, where $C_{i j}=X_{i} \cap X_{j}$ if $X_{i}$ and $X_{j}$ meet along a curve, or $C_{i j}=\emptyset$ otherwise (cf. Definition 2.5). Then:

$$
p_{\omega}(X)=h^{2}\left(G_{X}, \mathbb{C}\right)+\sum_{i=1}^{v} p_{g}\left(X_{i}\right)+\operatorname{dim}\left(\operatorname{coker}\left(\Phi_{X}\right)\right) .
$$

In particular, (1.1) shows that $p_{\omega}$ is a topological invariant of Zappatic surfaces.

Suppose now that a Zappatic surface $X$ is smoothable, namely $X=X_{0}$ is the central fibre of a flat degeneration $\pi: X \rightarrow \Delta$ of surfaces, where $\Delta$ is the spectrum of a DVR (or equivalently the complex unit disk) and each fibre $X_{t}=\pi^{-1}(t), 0 \neq t \in \Delta$, is smooth.

In [2], we gave sharp bounds for $K_{\mathcal{X}_{t}}^{2}, t \neq 0$; this enabled us to prove, in the above situation, a stronger version of the Miyaoka-Yau inequality for the general fiber $X_{t}$.

In this paper, we show that the $\omega$-genus of the fibres of a flat degeneration of surfaces with Zappatic central fibre as above is constant, namely we prove the following (cf. Theorem 4.14):

Theorem 2. Let $X \rightarrow \Delta$ be a flat degeneration of surfaces parametrized by a disc, such that the central fibre $X_{0}=X$ is Zappatic and each fibre $X_{t}, t \neq 0$, is smooth. Then, for any $t \neq 0$, one has:

$$
p_{g}\left(X_{t}\right)=p_{\omega}(X) .
$$

Let us briefly describe the contents of this paper. Section 2 recalls some basic definitions and notation concerning Zappatic surfaces. We associate to a Zappatic surface $X$ a dual graph $G_{X}$ which encodes the configuration of the irreducible components of $X$ as well as of its Zappatic singularities. For more details, the reader is referred to [1] and [2].

In Section 3 we compute the cohomology of the structure sheaf of a Zappatic surface $X$, thus proving Theorem 1 above, since $p_{\omega}(X)=h^{2}\left(X, \mathcal{O}_{X}\right)$. In order to do so, we exploit the natural injective resolution (3.4) of the sheaf $\mathcal{O}_{X}$ in terms of the structure sheaves of the irreducible components of $X$ and of its singular locus. An alternative, and in some sense dual, approach is via the interpretation of the global sections of $\omega_{X}$ as collections of meromorphic 2 -forms on the irreducible components of $X$, having poles along the double curves of $X$ with suitable matching conditions. This interpretation makes it possible, in principle, to compute $h^{0}\left(X, \omega_{X}\right)$ by computing the number of such independent collections of forms. This is the viewpoint taken in [1], where we discussed only the normal crossings case. However, the approach taken here leads more quickly and neatly to our result.

In Section 4 we consider flat degenerations $\pi: \mathcal{X} \rightarrow \Delta$, parametrized by a disc, of smooth surfaces to a Zappatic one $X=\mathcal{X}_{0}$ and we prove Theorem 2. We recall the construction of a normal crossing reduction $\bar{\pi}: \bar{X} \rightarrow \Delta$ of $\pi$, i.e. $\bar{X} \rightarrow X$ is a resolution of singularities of $X$ and the support of its central fibre $\bar{X}_{0}$ has global normal crossings (cf. Remark 4.2). Then we apply the results in Chapter II of [9] in order to get a semistable reduction $\tilde{\pi}: \tilde{X} \rightarrow \Delta$ of $\pi$. This enables us to deduce the topological properties of the fibres of $\tilde{X}$ from those of $X$, with the assistance of the Clemens-Schmid exact sequence (cf. e.g. [13]).

\section{Preliminaries}

In this paper we deal with projective varieties defined over the complex field $\mathbb{C}$.

Let us recall the notions of Zappatic singularities, Zappatic surfaces and their dual graphs. We refer the reader for more details to our previous papers [1] and [2]. One word of warning: 
what we call good Zappatic singularities there, here we simply call Zappatic singularities, because no other type of Zappatic singularity will be considered in this paper.

Definition 2.1. Let us denote by $R_{n}$ [resp. $S_{n}, E_{n}$ ] a graph which is a chain [resp. a fork, a cycle] with $n$ vertices, $n \geqslant 3$, cf. Figure 1 . Let $C_{R_{n}}\left[\right.$ resp. $\left.C_{S_{n}}, C_{E_{n}}\right]$ be a connected, projectively normal curve of degree $n$ in $\mathbb{P}^{n}$ [resp. in $\mathbb{P}^{n}$, in $\left.\mathbb{P}^{n-1}\right]$, which is a stick curve, i.e. a union of lines, whose dual graph is $R_{n}$ [resp. $S_{n}, E_{n}$.
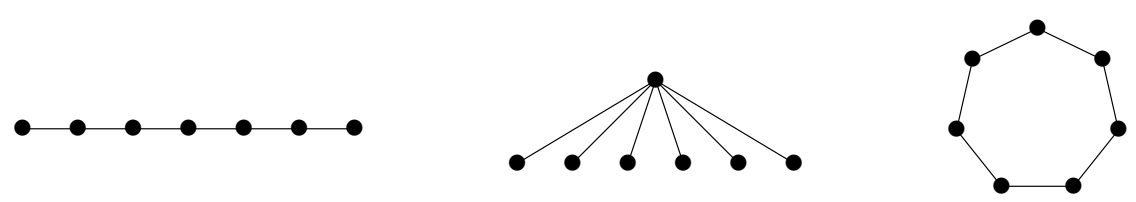

FiguRE 1. A chain $R_{n}$, a fork $S_{n}$ with $n-1$ teeth, a cycle $E_{n}$.

We say that a point $x$ of a projective surface $X$ is a $R_{n^{-}}$[resp. $S_{n^{-}}, E_{n^{-}}$] point if $(X, x)$ is locally analytically isomorphic to a pair $(Y, y)$ where $Y$ is the cone over a curve $C_{R_{n}}$ [resp. $\left.C_{S_{n}}, C_{E_{n}}\right], n \geqslant 3$, and $y$ is the vertex of the cone (cf. Figure 2). We say that $R_{n^{-}}, S_{n^{-}}$, $E_{n}$-points are Zappatic singularities.
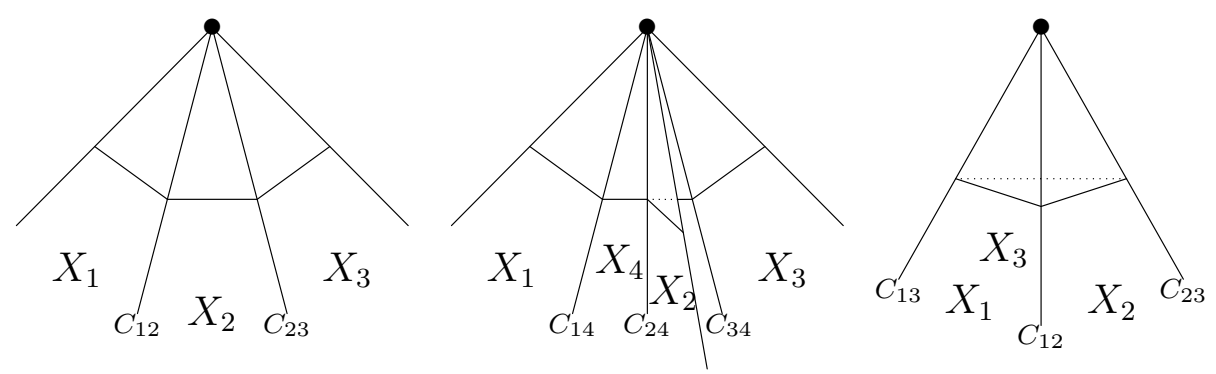

FiguRE 2. Examples: a $R_{3}$-point, a $S_{4}$-point and an $E_{3}$-point.

Definition 2.2. A projective surface $X=\bigcup_{i=1}^{v} X_{i}$ is called a Zappatic surface if $X$ is connected, reduced, all its irreducible components $X_{1}, \ldots, X_{v}$ are smooth and:

- the singularities in codimension one of $X$ are at most double curves which are smooth and irreducible along which two surfaces meet transversally;

- the further singularities of $X$ are Zappatic singularities.

We set $C_{i j}=X_{i} \cap X_{j}$ if $X_{i}$ and $X_{j}$ meet along a curve, we set $C_{i j}=\emptyset$ otherwise. We set $C_{i}=X_{i} \cap \overline{X-X_{i}}=\bigcup_{j=1}^{v} C_{i j}$. We denote by $C$ the singular locus of $X$, i.e. the curve $C=\bigcup_{1 \leqslant i<j \leqslant v} C_{i j}$.

Remark 2.3. A Zappatic surface $X$ is Cohen-Macaulay. Moreover it has global normal crossings except at the $R_{n^{-}}$and $S_{n}$-points, for $n \geqslant 3$, and at the $E_{m}$-points, for $m \geqslant 4$.

We associate to a Zappatic surface $X$ a dual graph $G_{X}$ as follows. Notice that this is slightly different from the graph defined in [1] and [2], which contains more information that we will not need here.

Definition 2.4. Let $X=\bigcup_{i=1}^{v} X_{i}$ be a Zappatic surface. The dual graph $G_{X}$ of $X$ is given by:

- a vertex $x_{i}$ for each irreducible component $X_{i}$ of $X$;

- an edge $l_{i j}$, joining the vertices $x_{i}$ and $x_{j}$, for each irreducible component of the curve $C_{i j}=X_{i} \cap X_{j}$; 
- a $n$-face $F_{p}$ for each point $p$ of $X$ of type $E_{n}$ for some $n \geqslant 3$ : the $n$ edges bounding the face $F_{p}$ are the $n$ irreducible components of the double curve $C$ of $X$ concurring at $p$.

We will denote by $\ell$ the number of edges and by $f$ the number of faces of $G_{X}$. We will put, once and for all, the lexicographic orientation on the edges of $G_{X}$, namely an index pair $i<j$, such that $C_{i j} \neq \emptyset$, corresponds to a set of edges in $G_{X}$ which are assumed to be oriented from $x_{i}$ to $x_{j}$. We will also fix an orientation for the faces of $G_{X}$.

By abusing notation, we will denote by $G_{X}$ also the natural CW-complex associated to the dual graph $G_{X}$ of $X$.

We now define the map $\Phi_{X}$ which appears in the statement of Theorem 1.

Definition 2.5. Let $X=\bigcup_{i=1}^{v} X_{i}$ be a Zappatic surface. Let $r_{i j}: H^{1}\left(X_{i}, \mathcal{O}_{X_{i}}\right) \rightarrow H^{1}\left(C_{i j}, \mathcal{O}_{C_{i j}}\right)$ be the restriction map to $C_{i j}$ as a divisor in $X_{i}$. We define the natural map:

$$
\Phi_{X}: \bigoplus_{i=1}^{v} H^{1}\left(X_{i}, \mathcal{O}_{X_{i}}\right) \rightarrow \bigoplus_{1 \leqslant i<j \leqslant v} H^{1}\left(C_{i j}, \mathcal{O}_{C_{i j}}\right), \quad \Phi_{X}\left(a_{i}\right)=-\sum_{j=1}^{i-1} r_{i j}\left(a_{i}\right)+\sum_{j=i+1}^{v} r_{i j}\left(a_{i}\right)
$$

if $a_{i} \in H^{1}\left(X_{i}, \mathcal{O}_{X_{i}}\right)$ and extend $\Phi_{X}$ linearly. When $X$ is clear from the context, we will write simply $\Phi$ instead of $\Phi_{X}$.

\section{The $\omega$-Genus of A ZAPPAtic SURface}

The aim of this section is to compute the $\omega$-genus of a Zappatic surface $X$. What we will actually do will be to compute the cohomology of the structure sheaf $\mathcal{O}_{X}$, which is sufficient, since $p_{\omega}(X)=h^{2}\left(X, \mathcal{O}_{X}\right)$.

The main result of this section (cf. Theorem 1 in the introduction) is the following:

Theorem 3.1. Let $X=\bigcup_{i=1}^{v} X_{i}$ be a Zappatic surface. Then:

$$
p_{\omega}(X)=h^{2}\left(X, \mathcal{O}_{X}\right)=h^{2}\left(G_{X}, \mathbb{C}\right)+\sum_{i=1}^{v} p_{g}\left(X_{i}\right)+\operatorname{dim}(\operatorname{coker}(\Phi)),
$$

and

$$
h^{1}\left(X, \omega_{X}\right)=h^{1}\left(X, \mathcal{O}_{X}\right)=h^{1}\left(G_{X}, \mathbb{C}\right)+\operatorname{dim}(\operatorname{ker}(\Phi))
$$

where $G_{X}$ is the dual graph of $X$ and $\Phi=\Phi_{X}$ is the map of Definition 2.5.

Proof. Let $p_{1}, \ldots, p_{f}$ be the $E_{n}$-points of $X, n \geqslant 3$. As in [1], proof of Proposition 3.15, one has the exact sequence:

$$
0 \rightarrow \mathcal{O}_{X} \rightarrow \bigoplus_{i=1}^{v} \mathcal{O}_{X_{i}} \stackrel{d_{G}^{0}}{\longrightarrow} \bigoplus_{1 \leqslant i<j \leqslant v} \mathcal{O}_{C_{i j}} \stackrel{d_{G}^{1}}{\longrightarrow} \bigoplus_{h=1}^{f} \mathcal{O}_{p_{h}} \rightarrow 0
$$

where the maps are defined as follows:

- The map $\mathcal{O}_{X} \rightarrow \oplus_{i=1}^{v} \mathcal{O}_{X_{i}}$ is the direct sum of the natural restriction maps.

- To define the map $d_{G}^{0}: \oplus_{i=1}^{v} \mathcal{O}_{X_{i}} \rightarrow \oplus_{1 \leqslant i<j \leqslant v} \mathcal{O}_{C_{i j}}$, we describe the composition of its restriction to each summand $\mathcal{O}_{X_{i}}$ with the projection to any summand $\mathcal{O}_{C_{h k}}$, with $h<k$. This map sends $g \in \mathcal{O}_{X_{i}}$ to:

(1) $0 \in \mathcal{O}_{C_{h k}}$, if both $h, k$ are different from $i$;

(2) $g_{\mid C_{i k}} \in \mathcal{O}_{C_{i k}}$ if $k>i$;

(3) $-g_{\mid C_{k i}} \in \mathcal{O}_{C_{k i}}$ if $k<i$; 
- To define the map $d_{G}^{1}: \oplus_{1 \leqslant i<j \leqslant v} \mathcal{O}_{C_{i j}} \rightarrow \oplus_{h=1}^{f} \mathcal{O}_{p_{h}}$ again we describe the composition of its restriction to each summand $\mathcal{O}_{C_{i j}}$, with $i<j$, with the projection to any summand $\mathcal{O}_{p_{h}}$. Suppose $p_{h}$ is an $E_{n}$-point corresponding to a face $F_{h}$ of $G_{X}$ such that $\partial F_{h}=\sum_{1 \leqslant i<j \leqslant v} e_{i j} C_{i j}$, where either $e_{i j}=0$ or $e_{i j}= \pm 1$. Then this map sends $g \in \mathcal{O}_{C_{i j}}$ to $e_{i j} g\left(p_{h}\right)$.

We note that the induced maps on global sections in each case are the corresponding cochain map for the graph $G_{X}$; this motivates the notation for these maps used in (3.4).

Let $\Lambda$ be the kernel of the sheaf map $d_{G}^{1}$, so that we have two short exact sequences

$$
0 \rightarrow \mathcal{O}_{X} \rightarrow \oplus_{i=1}^{v} \mathcal{O}_{X_{i}} \rightarrow \Lambda \rightarrow 0
$$

and

$$
0 \rightarrow \Lambda \rightarrow \oplus_{1 \leqslant i<j \leqslant v} \mathcal{O}_{C_{i j}} \stackrel{d_{G}^{1}}{\longrightarrow} \oplus_{h=1}^{f} \mathcal{O}_{p_{h}} \rightarrow 0 .
$$

The latter gives the long exact sequence:

$$
\begin{gathered}
0 \rightarrow H^{0}(\Lambda) \rightarrow \oplus_{1 \leqslant i<j \leqslant v} H^{0}\left(\mathcal{O}_{C_{i} j}\right) \stackrel{d_{G}^{1}}{\longrightarrow} \oplus_{h=1}^{f} H^{0}\left(\mathcal{O}_{p_{h}}\right) \rightarrow \\
\rightarrow H^{1}(\Lambda) \stackrel{\beta}{\longrightarrow} \oplus_{1 \leqslant i<j \leqslant v} H^{1}\left(\mathcal{O}_{C_{i j}}\right) \rightarrow 0
\end{gathered}
$$

and since the cokernel of the map $d_{G}^{1}$ is $H^{2}\left(G_{X}, \mathbb{C}\right)$, we derive the short exact sequence

$$
0 \rightarrow H^{2}\left(G_{X}, \mathbb{C}\right) \rightarrow H^{1}(\Lambda) \stackrel{\beta}{\longrightarrow} \oplus_{1 \leqslant i<j \leqslant v} H^{1}\left(\mathcal{O}_{C_{i j}}\right) \rightarrow 0
$$

From the short exact sequence (3.5) we have the long exact sequence:

$$
\begin{gathered}
0 \rightarrow H^{0}\left(\mathcal{O}_{X}\right) \rightarrow \oplus_{i=1}^{v} H^{0}\left(\mathcal{O}_{X_{i}}\right) \rightarrow H^{0}(\Lambda) \rightarrow \\
\rightarrow H^{1}\left(\mathcal{O}_{X}\right) \rightarrow \oplus_{i=1}^{v} H^{1}\left(\mathcal{O}_{X_{i}}\right) \stackrel{\alpha}{\longrightarrow} H^{1}(\Lambda) \rightarrow H^{2}\left(\mathcal{O}_{X}\right) \rightarrow \oplus_{i=1}^{v} H^{2}\left(\mathcal{O}_{X_{i}}\right) \rightarrow 0 .
\end{gathered}
$$

Now $H^{1}\left(G_{X}, \mathbb{C}\right.$ ) is the kernel of $d_{G}^{1}$ (which is $H^{0}(\Lambda)$ ) modulo the image of $d_{G}^{0}$, which is the image of the map $\oplus_{i=1}^{v} H^{0}\left(\mathcal{O}_{X_{i}}\right) \rightarrow H^{0}(\Lambda)$ in the first line above. Hence we recognize $H^{1}\left(G_{X}, \mathbb{C}\right)$ as the cokernel of this map, and therefore the second line of the above sequence becomes

$$
0 \rightarrow H^{1}\left(G_{X}, \mathbb{C}\right) \rightarrow H^{1}\left(\mathcal{O}_{X}\right) \rightarrow \oplus_{i=1}^{v} H^{1}\left(\mathcal{O}_{X_{i}}\right) \stackrel{\alpha}{\longrightarrow} H^{1}(\Lambda) \rightarrow H^{2}\left(\mathcal{O}_{X}\right) \rightarrow \oplus_{i=1}^{v} H^{2}\left(\mathcal{O}_{X_{i}}\right) \rightarrow 0 .
$$

Now the composition of the map $\beta$ with the map $\alpha$ is exactly the map $\Phi$ : $\Phi=\beta \circ \alpha$. We claim that $\alpha$ and $\Phi$ have the same kernel, which by (3.7) is equivalent to having $\operatorname{Im}(\alpha) \cap H^{2}\left(G_{X}, \mathbb{C}\right)(=$ $\operatorname{ker}(\beta))=\{0\}$.

If we are able to show this, then the leftmost part of the above sequence would split off as

$$
0 \rightarrow H^{1}\left(G_{X}, \mathbb{C}\right) \rightarrow H^{1}\left(\mathcal{O}_{X}\right) \rightarrow \operatorname{ker}(\alpha)=\operatorname{ker}(\Phi) \rightarrow 0
$$

which would prove the $H^{1}$ statement of the theorem. In addition, if this is true, then the natural surjection from the cokernel of $\alpha$ to the cokernel of $\Phi$ would have $\operatorname{ker}(\beta)=H^{2}\left(G_{X}, \mathbb{C}\right)$ as its kernel, and we would have $\operatorname{dim}(\operatorname{coker}(\alpha))=\operatorname{dim} H^{2}\left(G_{X}, \mathbb{C}\right)+\operatorname{dim}(\operatorname{coker}(\Phi))$. Since the rightmost part of the long exact sequence above splits off as

$$
0 \rightarrow \operatorname{coker}(\alpha) \rightarrow H^{2}\left(\mathcal{O}_{X}\right) \rightarrow \oplus_{i=1}^{v} H^{2}\left(\mathcal{O}_{X_{i}}\right) \rightarrow 0
$$

we see that the $H^{2}$ statement of the theorem follows also.

To prove that $\operatorname{Im}(\alpha) \cap H^{2}\left(G_{X}, \mathbb{C}\right)=\{0\}$, notice that the sheaf map $d_{G}^{0}$ (which has $\Lambda$ as its image) factors through obvious maps:

$$
\oplus_{i=1}^{v} \mathcal{O}_{X_{i}} \rightarrow \oplus_{i=1}^{v} \mathcal{O}_{C_{i}} \rightarrow \oplus_{1 \leqslant i<j \leqslant v} \mathcal{O}_{C_{i j}}
$$

and therefore the map $\alpha$ on the $H^{1}$ level factors as:

$$
\oplus_{i=1}^{v} H^{1}\left(\mathcal{O}_{X_{i}}\right) \rightarrow \oplus_{i=1}^{v} H^{1}\left(\mathcal{O}_{C_{i}}\right) \rightarrow H^{1}(\Lambda) .
$$


Moreover one has the short exact sequence:

$$
0 \rightarrow \mathcal{O}_{C} \rightarrow \oplus_{i=1}^{v} \mathcal{O}_{C_{i}} \rightarrow \Lambda \rightarrow 0
$$

where $C$ is the singular locus of $X$, and thus we have an exact sequence:

$$
H^{1}\left(C, \mathcal{O}_{C}\right) \rightarrow \oplus_{i=1}^{v} H^{1}\left(\mathcal{O}_{C_{i}}\right) \rightarrow H^{1}(\Lambda) \rightarrow 0 .
$$

We remark now that $H^{1}\left(C_{i}, \mathcal{O}_{C_{i}}\right)$ [resp. $H^{1}\left(C, \mathcal{O}_{C}\right)$ ] is the tangent space at the origin to $\operatorname{Pic}^{0}\left(C_{i}\right)$ [resp. to $\mathrm{Pic}^{0}(C)$ ] which is a $\left(\mathbb{C}^{*}\right)^{\delta_{i}}$-extension [resp. a $\left(\mathbb{C}^{*}\right)^{\delta}$-extension] of $\oplus_{j=1}^{v} \operatorname{Pic}^{0}\left(C_{i j}\right)$ [resp. of $\oplus_{1 \leqslant i<j \leqslant v} \operatorname{Pic}^{0}\left(C_{i j}\right)$ ], where $\delta_{i}$ [resp. $\delta$ ] depends on the singular points of $C_{i}$ [resp. of $C$.

There are natural restriction maps:

$$
a: \oplus_{i=1}^{v} \operatorname{Pic}^{0}\left(X_{i}\right) \rightarrow \oplus_{i=1}^{v} \operatorname{Pic}^{0}\left(C_{i}\right)
$$

and

$$
b: \operatorname{Pic}^{0}(C) \rightarrow \oplus_{i=1}^{v} \operatorname{Pic}^{0}\left(C_{i}\right)
$$

which are maps of $\mathbb{C}^{*}$-extensions of abelian varieties; their differentials at the origin are

$$
\oplus_{i=1}^{v} H^{1}\left(\mathcal{O}_{X_{i}}\right) \rightarrow \oplus_{i=1}^{v} H^{1}\left(\mathcal{O}_{C_{i}}\right)
$$

and

$$
H^{1}\left(C, \mathcal{O}_{C}\right) \rightarrow \oplus_{i=1}^{v} H^{1}\left(\mathcal{O}_{C_{i}}\right)
$$

respectively; the latter is the leftmost map of the sequence (3.8).

The map $b$ appears in the following exact diagram:

$$
\begin{aligned}
& 0 \rightarrow\left(\mathbb{C}^{*}\right)^{\delta} \quad \rightarrow \quad \operatorname{Pic}^{0}(C) \quad \rightarrow \quad \oplus_{i<j} \operatorname{Pic}^{0}\left(C_{i j}\right) \rightarrow 0 \\
& 0 \rightarrow \oplus_{i}\left(\mathbb{C}^{*}\right)^{\delta_{i}} \rightarrow \oplus_{i} \operatorname{Pic}^{0}\left(C_{i}\right) \rightarrow \oplus_{i, j} \operatorname{Pic}^{0}\left(C_{i j}\right) \rightarrow 0
\end{aligned}
$$

The vertical map on the right is an injection; indeed, it is the direct sum of diagonal maps $\operatorname{Pic}^{0}\left(C_{i j}\right) \rightarrow \operatorname{Pic}^{0}\left(C_{i j}\right) \oplus \operatorname{Pic}^{0}\left(C_{j i}\right)$. Therefore, if we denote by $V$ the cokernel of the central map $b$, we have a short exact sequence of cokernels

$$
0 \rightarrow\left(\mathbb{C}^{*}\right)^{\gamma} \rightarrow V \rightarrow \oplus_{i<j} \operatorname{Pic}^{0}\left(C_{i j}\right) \rightarrow 0
$$

for some $\gamma$; in particular, $\mathrm{V}$ is again a $\mathbb{C}^{*}$-extension of abelian varieties. We now recognize by $(3.8)$ that $H^{1}(X, \Lambda)$ is the tangent space at the origin to $V$; moreover the sequence (3.7) is the map on tangent spaces for the above sequence of groups. In particular the map $\beta$ is the tangent space map for the projection $V \rightarrow \oplus_{i<j} \operatorname{Pic}^{0}\left(C_{i j}\right)$.

Composing $a$ with the projection of $\oplus_{i=1}^{v} \operatorname{Pic}^{0}\left(C_{i}\right)$ to $V$ gives a map

$$
c: \oplus_{i=1}^{v} \operatorname{Pic}^{0}\left(X_{i}\right) \rightarrow V
$$

whose differential at the origin is the previously encountered map

$$
\alpha: \oplus_{i=1}^{v} H^{1}\left(\mathcal{O}_{X_{i}}\right) \rightarrow H^{1}(X, \Lambda) .
$$

Now $\oplus_{i=1}^{v} \operatorname{Pic}^{0}\left(X_{i}\right)$ is compact, and therefore the image of $c$ in $V$ has finite intersection with the kernel of the projection $V \rightarrow \oplus_{1 \leqslant i<j \leqslant v} \operatorname{Pic}^{0}\left(C_{i j}\right)$. At the tangent space level, this means that the image of $\alpha$ has trivial intersection with the kernel of the map $\beta$, which we have identified as $H^{2}\left(G_{X}, \mathbb{C}\right)$, which was to be proved.

Remark 3.9. Note that the formulas (3.2) and (3.3) agree with, and imply, the formula:

$$
\chi\left(\mathcal{O}_{X}\right)=\sum_{i=1}^{v} \chi\left(\mathcal{O}_{X_{i}}\right)-\sum_{1 \leqslant i<j \leqslant v} \chi\left(\mathcal{O}_{C_{i j}}\right)+f
$$

we found in [1], Proposition 3.15. 


\section{Degenerations to Zappatic surfaces}

In this section we focus on degenerations of smooth surfaces to Zappatic ones.

Definition 4.1. Let $\Delta$ be the spectrum of a DVR (equiv. the complex unit disk). A degeneration of surfaces parametrized by $\Delta$ is a proper and flat morphism $\pi: \mathcal{X} \rightarrow \Delta$ such that each fibre $X_{t}=\pi^{-1}(t), t \neq 0$ (where 0 is the closed point of $\Delta$ ), is a smooth, irreducible, projective surface.

We will say that $X \rightarrow \Delta$ is a normal crossing degeneration if the total space $\mathcal{X}$ is smooth

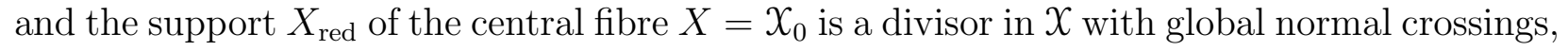
i.e. $X_{\text {red }}$ is a Zappatic surface with only $E_{3}$-points as Zappatic singularities.

A normal crossing degeneration is called semistable if the central fibre is reduced.

Remark 4.2. Given a degeneration $\pi: X \rightarrow \Delta$, Hironaka's Theorem on the resolution of singularities implies that there exists a birational morphism $\bar{X} \rightarrow X$ such that $\bar{X} \rightarrow \Delta$ is a normal crossing degeneration, which we will call a normal crossing reduction of $\pi$.

Given a degeneration $\pi: \mathcal{X} \rightarrow \Delta$, the Semistable Reduction Theorem (see Theorem on p. 53-54 in [9]) states that there exists a base change $\beta: \Delta \rightarrow \Delta$, defined by $\beta(t)=t^{m}$, for some $m$, a semistable degeneration $\tilde{\pi}: \tilde{X} \rightarrow \Delta$ and a diagram

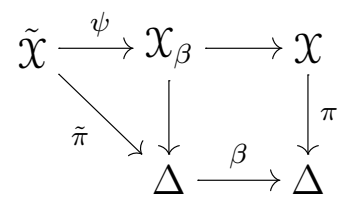

such that the square is Cartesian and $\psi: \tilde{X} \rightarrow X_{\beta}$ is a birational morphism obtained by blowing-up a suitable sheaf of ideals on $X_{\beta}$. This is called a semistable reduction of $\pi$.

The geometric genus of the general fibre of a semistable degeneration of surfaces can be computed via the Clemens-Schmid exact sequence, cf. [13]. Clemens-Schmid result implies the following:

Theorem 4.4. Let $X=\bigcup_{i=1}^{v} X_{i}$ be the central fibre of a semistable degeneration of surfaces $X \rightarrow \Delta$. Let $G_{X}$ be the dual graph of $X$ and $\Phi_{X}$ be the map introduced in Definition 2.5. Then, for $t \neq 0$, one has:

$$
p_{g}\left(X_{t}\right)=h^{2}\left(G_{X}, \mathbb{C}\right)+\sum_{i=1}^{v} p_{g}\left(X_{i}\right)+\operatorname{dim}\left(\operatorname{coker}\left(\Phi_{X}\right)\right) .
$$

Then Theorem 4.4 and our Theorem 3.1 imply the following:

Corollary 4.6. Let $X \rightarrow \Delta$ be a semistable degeneration of surfaces, so that its central fibre $X=X_{0}$ is a Zappatic surface with only $E_{3}$-points as Zappatic singularities. Then, for any $t \neq 0$, one has:

$$
p_{g}\left(X_{t}\right)=p_{\omega}(X) .
$$

Remark 4.7. Let $X \rightarrow \Delta$ be a degeneration of surfaces with central fibre $X$. Consider the dualizing sheaf $\omega_{X}$ of $X$. By general properties of dualizing sheaves, one knows that $\omega_{X}$ is torsion-free as an $\mathcal{O}_{x}$-module. Since one has the injection $\mathcal{O}_{\Delta} \hookrightarrow \mathcal{O}_{x}$, then $\omega_{x}$ is torsion-free over $\Delta$. Since $\Delta$ is the spectrum of a DVR, then $\omega_{x}$ is free and therefore flat over $\Delta$. By semi-continuity, this implies that, for $t \neq 0, p_{g}\left(X_{t}\right) \leqslant p_{\omega}(X)$. The above corollary shows that equality holds for semistable degenerations of surfaces.

Consider, from now on, a degeneration $\pi: \mathcal{X} \rightarrow \Delta$ of surfaces with Zappatic central fibre $X=X_{0}$. Our main purpose in this section is to prove Proposition 4.12 , where we show that the $\omega$-genus of the central fibre of a semistable reduction $\tilde{\pi}: \tilde{X} \rightarrow \Delta$ of $\pi$ equals the $\omega$-genus 
of $X$. As a consequence we will have that the $\omega$-genus of the fibres of $\pi: \mathcal{X} \rightarrow \Delta$ is constant (see Theorem 4.14 below), exactly as it happens in the normal crossings case, as we saw in Corollary 4.6. In order to prove Proposition 4.12, it is necessary to carefully analyze the process, basically described in Chapter II of [9], which produces the semistable reduction.

As we said, Hironaka's result implies the existence of a normal crossing reduction of $\pi$. The birational transformation involved in resolving the singularities can be taken to be a sequence of blow-ups (which one can arrange to be at isolated points and along smooth curves) interspersed with normalization maps. For general singularities such a procedure may introduce components and double curves which affect the $\omega$-genus of the central fibre. Our next task is to show that, under the assumption that the central fibre is Zappatic, we have very precise control over the $\omega$-genus. For this we will need to more explicitly describe an algorithm which produces a resolution. In order to do this, we will use, as common in programming languages, the word "while" to indicate that the statement following it is repeated until it becomes false.

Normal crossing reduction algorithm 4.8. Let $X \rightarrow \Delta$ be a degeneration of surfaces with Zappatic central fibre.

Step 1: while $\mathcal{X}_{0}$ has a point $p$ of type either $R_{n}$ or $S_{n}, n \geqslant 3$, replace $\mathcal{X}$ by its blow-up at $p$; Step 2: while $X_{0}$ has a point $p$ of type $E_{n}$ and $X$ has multiplicity $n \geqslant 3$ at $p$, replace $\mathcal{X}$ by its blow-up at $p$

Step 3: while $\mathcal{X}$ has a double curve $\gamma$, replace $\mathcal{X}$ by its blow-up along $\gamma$;

Step 4: if $\mathcal{X}$ has a double point $p$, then replace $\mathcal{X}$ by the normalization of its blow-up at $p$ and go back to Step 3;

Step 5: while there is a component of $\mathcal{X}_{0}$ with a double point $p$, replace $\mathcal{X}$ by its blow-up at $p$

Step 6: while there are two components $X_{1}$ and $X_{2}$ of $X_{0}$ meeting along a curve with a node $p$, first blow-up $X$ at $p$, then blow-up along the line which is the intersection of the exceptional divisor with the proper transform of $\mathcal{X}_{0}$, and finally replace $\mathcal{X}$ with the resulting threefold.

The following proposition is devoted to prove that this algorithm works.

Proposition 4.9. Let $\pi: X \rightarrow \Delta$ be a degeneration of surfaces with Zappatic central fibre $X=X_{0}=\bigcup_{i=1}^{v} X_{i}$ and run the normal crossing reduction algorithm 4.8. The algorithm stops after finitely many steps and its output gives a normal crossing reduction $\bar{\pi}: \bar{X} \rightarrow \Delta$ of $\pi$.

Proof. The total space $\mathcal{X}$ of $\pi$ may have the following singularities:

- double curves, which are double curves also for $X$;

- isolated double points along the double curves of $X$;

- further singular points at the Zappatic singularities of $X$, which can be isolated or may occur on double curves of the total space.

Our aim is to prove that the normal crossing reduction algorithm 4.8 resolves the singularities of the total space and produces a central fibre whose support has global normal crossings.

(Step 1) By Proposition 5.17 in [2], if $X$ has either a $R_{n}$-point or a $S_{n}$-point, $n \geqslant 3$, then the total space $X$ has multiplicity $n$ at $p$. Let $X^{\prime} \rightarrow X$ be the blow-up of $X$ at a $R_{n}$-point [resp. $S_{n}$-point] $p$. By Proposition 5.23 in [2], the exceptional divisor $E$ is a Zappatic surface of degree $n$ in $\mathbb{P}^{n+1}$ such that all of its irreducible components are rational normal surfaces meeting along lines and $E$ has at most $R_{m}$-points, $m \leqslant n$ [resp. $S_{m}$-points, $m \leqslant n$ ] as Zappatic singularities. Let $X^{\prime}$ be the proper transform of $X$. The curve $\Gamma=E \cap X^{\prime}$ is a stick curve $C_{R_{n}}$ [resp. $C_{S_{n}}$ ] which, being nodal, does not contain any Zappatic singularity of $E$. The new central fibre $E \cup X^{\prime}$ has either $E_{3^{-}}$or $E_{4}$-points at the double points of $\Gamma$, depending on 
whether $E$ is smooth or has a double point there. These points are accordingly either smooth or double points for $X^{\prime}$.

The fact that Step 1 is repeated finitely many times follows e.g. from Proposition 3.4.13 in [10]. If $X^{\prime} \rightarrow X$ is the composition of all the blow-ups done in Step 1 , then $X^{\prime} \rightarrow \Delta$ is a degeneration whose central fibre is a Zappatic surface with only $E_{n}$-points, $n \geqslant 3$, as Zappatic singularities.

(Step 2) By Proposition 5.17 in [2], if $X$ has an $E_{n}$-point $p, n \geqslant 3$, then either $X$ has multiplicity $n$ at $p$, or $n \leqslant 4$ and $\mathcal{X}$ has at most a double point at $p$. In this step we consider only the former possibility, since the other cases are considered in the next steps. Let $X^{\prime} \rightarrow X$ be the blow-up of $\mathcal{X}$ at $p$. By Proposition 5.23 of [2], as shown in the proof of Theorem 7.2 therein, the exceptional divisor $E$ is a Gorenstein surface of degree $n$ in $\mathbb{P}^{n}$ which is one of the following:

(I) an irreducible del Pezzo surface, possible only if $n \leqslant 6$;

(II) a union $F=F_{1} \cup F_{2}$ of two irreducible components $F_{1}$ and $F_{2}$ such that $F_{1} \cap F_{2}$ is a (possibly reducible) conic; the surface $F_{i}, i=1,2$, is either a smooth rational normal cubic scroll, or a quadric, or a plane;

(III) a Zappatic surface, whose $m \leqslant n$ irreducible components meet along lines and are either planes or smooth quadrics; moreover $E$ has a unique Zappatic singularity, which is an $E_{m}$-point.

In case (I), the del Pezzo surface $E$ has at most isolated rational double points.

In case (II), the surface $E$ is Zappatic unless either the conic is reducible or one of the two components is a quadric cone. Note that, if $F_{1} \cap F_{2}$ is a conic with a double point $p^{\prime}$, then $F_{1}$ and $F_{2}$ are tangent at $p^{\prime}$ and $E$ has not normal crossings.

Let $X^{\prime}$ be the proper transform of $X$. The curve $\Gamma=E \cap X^{\prime}$ is a stick curve $C_{E_{n}}$. In case (II), if an irreducible component of $E$ is a quadric cone, the vertex of the cone is a double point of $\Gamma$ and $X^{\prime}$ also has a double point there. In case (III), the curve $\Gamma$, being nodal, does not contain the $E_{m}$-point of $E$. As in Step 1, one sees that the singular points of $\Gamma$ are either smooth or double points for $X^{\prime}$.

In cases (I) and (II), we have eliminated the original Zappatic $E_{n}$ singularity; in case (III), we have a single $E_{m}(m \leqslant n)$ point to still consider. Whatever extra double points have been introduced, will be handled in later steps.

As Step 1, also Step 2 is repeated finitely many times e.g. by Proposition 3.4.13 in [10].

(Step 3) Now the total space $\mathcal{X}$ of the degeneration has at most double points. Suppose that $\mathcal{X}$ is singular in dimension one and let $\gamma$ be an irreducible curve which is double for $X$. Then $\gamma$ lies in the intersection of two irreducible components $X_{1}$ and $X_{2}$ of $X$. By Definition 2.1 of Zappatic surface and the previous steps (cf. Sections 6 and 7 in [2]), one has that $\gamma$ is smooth and the intersection of $X_{1}$ and $X_{2}$ is transversal at the general point of $\gamma$.

Now let $X^{\prime} \rightarrow X$ be the blow-up of $X$ along $\gamma$. Let $E$ be the exceptional divisor and $X_{i}^{\prime}$, $i=1,2$, be the proper transform of $X_{i}$ in $X^{\prime}$. Let $p$ be the general point of $\gamma$. Note that there are effective Cartier divisors of $X$ through $p$ having a node at $p$. Therefore there are effective Cartier divisors of $\mathcal{X}$ through $p$ having at $p$ a double point of type $A_{k}$, for some $k \geqslant 1$. Since the exceptional divisor of a minimal resolution of such a point does not contain multiple components, we see that $E$ must be reduced. Then $E$ is a conic bundle and $\gamma_{i}=E \cap X_{i}^{\prime}$, $i=1,2$, is a section of $E$ isomorphic to $\gamma$.

Let $C$ be the general ruling of $E$. If $C$ is irreducible, then $E$ is irreducible and has at most isolated double points. We remark moreover that $\gamma_{1}$ and $\gamma_{2}$ are generically smooth for the total space $X^{\prime}$, since they are generically smooth for $E$, which is a Cartier divisor of $X^{\prime}$. In this case, we got rid of the double curve. 
Let $C=r_{1} \cup r_{2}$ be reducible into two distinct lines. We may assume that $r_{i} \cap \gamma_{i}, i=1,2$, is a point whereas $r_{i} \cap \gamma_{3-i}=\emptyset$. This implies that $E$ is reducible; one component meets $X_{1}^{\prime}$ and the other meets $X_{2}^{\prime}$. Hence we may write $E=F_{1} \cup F_{2}$, where $F_{i}$ meets generically transversally $X_{i}^{\prime}$ along $\gamma_{i}, i=1,2$. It may happen that $F_{1}$ and $F_{2}$ meet, generically transversally, along finitely many fibres of their rulings; away from these, they meet along the curve $\gamma^{\prime}$, whose general point is $r_{1} \cap r_{2}$.

We note that $\gamma^{\prime}$, being isomorphic to $\gamma$, is smooth. Moreover, a local computation shows that $F_{1}$ and $F_{2}$ meet transversally at a general point of $\gamma^{\prime}$. If the general point of $\gamma^{\prime}$ is smooth for $X^{\prime}$, we have nothing to do with $\gamma^{\prime}$, otherwise we go on blowing-up $X^{\prime}$ along $\gamma^{\prime}$. As usual, after finitely many blow-ups we get rid of all the curves which are double for the total space.

(Step 4) Now the total space $X$ of the degeneration has at most isolated double points. Let $X_{\text {red }}$ be the support of the central fibre $X$. Note that, the first time one reaches this step, one has that $X_{\text {red }}=X$, which implies that $X_{\text {red }}$ is Cartier. In what follows, we only require that in a neighborhood of the singular points where we apply this step, the reduced set of components is Cartier.

By the discussion of the previous steps, one sees that a double point $p$ of $X$ can be of the following types (cf. Figure 3):

(a) an isolated double point of $X_{\text {red }}$;

(b) a point of a double curve of $X_{\text {red }}$;

(c) an $E_{3}$-point of $X_{\text {red }}$;

(d) an $E_{4}$-point of $X_{\text {red }}$;

(e) a quadruple point of $X_{\text {red }}$ which lies in the intersection of three irreducible components $X_{1}, X_{2}$ and $X_{3}$ of $X_{\text {red }}$; two of them, say $X_{2}$ and $X_{3}$, are smooth at $p$, whereas $X_{1}$ has a rational double point of type $A_{k}, k \geqslant 1$, at $p$. In this case, $X_{2} \cup X_{3}$ and $X_{1}$ are both complete intersection of $\mathcal{X}$ locally at $p$.

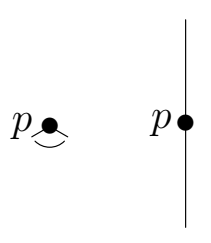

(a)

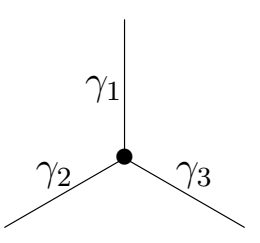

(c)

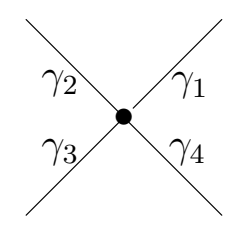

$(d)$

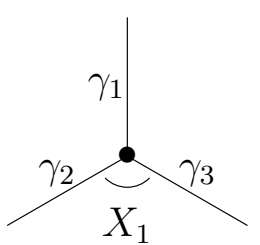

$(e)$

Figure 3. Types of double points of the total space $X$.

Double points of type (a) may appear either in Step 2, if the exceptional divisor is a singular del Pezzo surface, or in Step 3, if the exceptional divisor is a singular conic bundle. In both cases, they are rational double points for $X_{\text {red }}$. By resolving them, one clearly gets as exceptional divisors only rational surfaces meeting each other (and the proper transform of the central fibre) along rational curves.

Consider a double point $p$ of type (b), so $p$ lies on a double curve which is in the intersection of two irreducible components $X_{1}$ and $X_{2}$ of $X_{\text {red }}$. Let $X^{\prime} \rightarrow X$ be the blow-up of $X$ at $p$ and let $E$ be the exceptional divisor, which is a quadric surface in $\mathbb{P}^{3}$. Denote by $X_{i}^{\prime}$ the proper transform of $X_{i}, i=1,2$, and by $p^{\prime}$ the point $p^{\prime}=E \cap X_{1}^{\prime} \cap X_{2}^{\prime}$. Since a general hyperplane section of $X_{1} \cup X_{2}$ at $p$ is a curve with a node at $p$, the quadric $E$ is either:

(i) a smooth quadric meeting $X_{i}^{\prime}, i=1,2$, along a line; or

(ii) an irreducible quadric cone with vertex $p^{\prime}$; or

(iii) the union of two distinct planes meeting along a line $\gamma$ passing through $p^{\prime}$. 
In case (i), we resolved the singularity of the total space at $p$. In case (ii), the new total space $X^{\prime}$ has an isolated double point of type (e) at $p^{\prime}$. In case (iii), there are two possibilities: if the line $\gamma$ is a double curve of $X^{\prime}$, then we go back to Step 3, otherwise $X^{\prime}$ has an isolated double point of type $(\mathrm{d})$.

Let $p$ be a double point of type (c). According to Proposition 5.17 in [2], the embedding dimension of $\mathcal{X}$ at $p$ is 4 and the central fibre is locally analytically near $p$ a hyperplane section of $\mathcal{X}$. Since the multiplicity of the singularity of the threefold is two, and the multiplicity of the central fibre at this point is three, the locally analytic hyperplane section must contain a component of the tangent cone of the threefold singularity. This tangent cone is therefore a quadric which has rank at most two: it is either two distinct hyperplanes or a double hyperplane (i.e. a hyperplane counted twice). In fact a local computation shows that the latter cannot happen. In the former case, when one blows up $\mathcal{X}$ at $p$, one introduces two planes in the new central fibre. One of these planes meets the proper transforms of the three components each in a line, forming a triangle in that plane; this plane is double in the new central fibre. (Note that at this point we introduce a non-reduced component of the central fibre; but the rest of the algorithm does not involve this multiple component.) The other of the planes, which is simple in the new central fibre, meets each of the proper transforms at a single distinct point, which is still an ordinary double point of the total space. Three more blow-ups, one each at these double points, locally resolve the total space. (This analysis follows from a local computation.)

Consider now a double point $p$ of type (d). By Proposition 5.17 in [2], locally the tangent cone of $X$ at $p$ is a quadric cone in $\mathbb{P}^{3}$ and the tangent cone $T$ of $X_{\text {red }}$ at $p$ is obtained by cutting it with another quadric cone in $\mathbb{P}^{3}$, hence $T$ is a cone in $\mathbb{P}^{4}$ over a reduced, projectively normal curve of degree 4 and arithmetic genus 1 which spans a $\mathbb{P}^{3}$. Let $X^{\prime} \rightarrow X$ be the blowup of $X$ at $p$ and let $E$ be the exceptional divisor. Then $E$ is a quadric meeting the proper transform of $X$ along a stick curve $C_{E_{4}}$, therefore $E$ is either

(i) a smooth quadric; or

(ii) the union of two distinct planes meeting along a line $\gamma$.

In case (i), we resolved the singularity of $\mathcal{X}$ at $p$. In case (ii), there are two possibilities: if the line $\gamma$ is a double curve of $X^{\prime}$, then we go back to Step 3, otherwise $X^{\prime}$ has again two isolated double points of type (d) at the intersection of $\gamma$ with the proper transform of $X_{\text {red }}$.

Here we have created double components of the central fibre, namely the exceptional divisor is counted twice. However this exceptional divisor is a Cartier divisor, and therefore $X_{\text {red }}$ is also a Cartier divisor locally near this exceptional divisor.

Finally let $p=X_{1} \cap X_{2} \cap X_{3}$ be a double point of type (e). As in the case of type (d), locally the tangent cone of $X$ at $p$ is a quadric cone in $\mathbb{P}^{3}$, whereas the tangent cone $T$ of $X_{\text {red }}$ at $p$ is a cone in $\mathbb{P}^{4}$ over a reduced, projectively normal curve of degree 4 and arithmetic genus 1 which spans a $\mathbb{P}^{3}$. Let $X^{\prime} \rightarrow X$ be the blow-up of $X$ at $p$ and let $E$ the exceptional divisor. Denote by $p^{\prime}$ the intersection of $E$ with the proper transform of $X_{2}$ and $X_{3}$. Then $E$ is a quadric meeting the proper transform of $X_{\text {red }}$ along the union of two lines and a conic spanning a $\mathbb{P}^{3}$, therefore $E$ is either

(i) a smooth quadric; or

(ii) a quadric cone with vertex at $p^{\prime}$; or

(iii) a pair of planes.

In case (i), we resolved the singularity of the total space at $p$. In case (ii), the total space $X^{\prime}$ has at $p^{\prime}$ again a point of type (e). More precisely, if $p$ is a rational double point of type $A_{k}$, then $p^{\prime}$ is a rational double point of type $A_{k-1}$ for $E$. In case (iii), the line of intersection of the two planes may be singular for the new total space; if so, we return to Step 3. If not, there are again isolated double points of type $(d)$ and we iterate this step again. 
As in the case of type (d), the reduced central fibre remains Cartier in a neighborhood of the new exceptional locus.

It is clear that, after having repeated finitely many times Steps 3 and 4, one resolves the singularities of the total space at the double points of these five types (a)-(e).

We remark that we can proceed, in Step 4, by first resolving all of the points of type (c), and that such points are not created in the resolutions of points of type (d) and (e). In fact they are not created in any later step of the algorithm. Indeed, anytime three components $X_{1}, X_{2}$, and $X_{3}$ concur at a point as in type (c) where at least one of the three surfaces has been created by blowing-up, we claim that exactly one of the three surfaces has been created by blowing-up (i.e., is an exceptional divisor). Since such an exceptional divisor is locally Cartier and smooth at the point, then the total space is smooth at the point and therefore the point cannot be of type (c). To prove the claim, note that the only other possibility is that two of the three components, say $X_{2}$ and $X_{3}$ belong to an exceptional divisor. By blowing them down, then $X_{1}$ acquires a singular point which is worse than an ordinary double point, which is impossible.

(Step 5) Let $p$ be an isolated double point of the central fibre $X$ which is a smooth point of $X$. According to the previous steps, $p$ is either a rational double point of a del Pezzo surface or the singular point of a reduced fibre of a conic bundle. In both cases, the singularity of $X$ at $p$ is resolved by finitely many blow-ups. Since $p$ is a smooth point of $\mathcal{X}$, the exceptional divisor of each blow-up is a plane.

(Step 6) Following the previous steps, one sees that the support of the central fibre $X$ has global normal crossings, except at the points $p$, where two components $X_{1}$ and $X_{2}$ of $X$ meet along a curve with a node at $p$. Note that $X_{1}$ and $X_{2}$ are indeed tangent at $p$.

If one blows-up $X$ at $p$, the exceptional divisor $E$ is a plane meeting the proper transform $X_{i}^{\prime}$ of $X_{i}, i=1,2$, along a line $\gamma$, which is a $(-1)$-curve both on $X_{1}^{\prime}$ and $X_{2}^{\prime}$. The support of the new central fibre has not yet normal crossings. However a further blow-up along $\gamma$ produces the normal crossing reduction.

Let $X=\bigcup_{i=1}^{v} X_{i}$ be the central fibre of the original degeneration and let $\bar{X}_{\text {red }}=\bigcup_{i=1}^{w} \bar{X}_{i}$ be the support of the central fibre $\bar{X}$ of its normal crossing reduction obtained as above, where $w \geqslant v$. Next we describe the relation between the dual graph $G$ of $X$ and the one $\bar{G}$ of $\bar{X}_{\text {red }}$. By the proof of Proposition 4.8, one has that $G$ is a subgraph of $\bar{G}$ and we may assume that $\bar{X}_{i}$ is birational to $X_{i}, i=1, \ldots, v$.

Proposition 4.10. In the above situation, one has:

(i) $p_{g}\left(\bar{X}_{i}\right)=0, i=v+1, \ldots, w$;

(ii) $\operatorname{dim}\left(\operatorname{coker}\left(\Phi_{\bar{X}_{\text {red }}}\right)\right)=\operatorname{dim}\left(\operatorname{coker}\left(\Phi_{X}\right)\right)$;

(iii) the graphs $G$ and $\bar{G}$ have the same Betti numbers.

Proof. Following the discussion of each Step of the normal crossing reduction algorithm 4.8, one sees that each new component $\bar{X}_{i}, i=v+1, \ldots, w$, of the central fibre is an exceptional divisor of a blow-up, which is either a rational or a ruled surface. This proves (i).

For $i=1, \ldots, v$, the birational morphism $\bar{\sigma}: \bar{X} \rightarrow X$ determines a birational morphism $\bar{X}_{i} \rightarrow X_{i}$ which is the composition of blow-ups at smooth points of $X_{i}$. In order to prove (ii), we notice that in algorithm 4.8, we have added rational double curves (which do not contribute to the cokernel), new rational components (which also do not contribute to the cokernel), and irrational ruled surfaces, which are only created by blowing-up irrational double curves. Focusing on single such irrational double curve, one sees that it is replaced by a certain number $h$ of irrational ruled surfaces, and by $h+1$ new double curves. The map on the $H^{1}$ 
level is an isomorphism between the new surfaces and the new curves. Hence there is no change in the dimension of the cokernel. This concludes the proof of (ii).

In order to prove (iii), let us see what happens at each step of algorithm 4.8.

In Step 1 one blows-up $R_{n^{-}}$and $S_{n}$-points of $X=X_{0}$. An example will illustrate the key features of the analysis. Let $p$ be a $R_{4}$-point of $X$. After blowing-up $\mathcal{X}$ at $p$, there are five different possible configurations of the exceptional divisor $E$ (cf. the proof of Claim 6.20 in $[2]):$

(i) $E$ is the union of two quadrics with normal crossings;

(ii) $E$ is the union of a quadric and two planes having a $R_{3}$-point $p^{\prime}$, and the quadric is in the middle;

(iii) $E$ is the union of a quadric and two planes having a $R_{3}$-point $p^{\prime}$, and one of the planes is in the middle;

(iv) $E$ is the union of four planes having two $R_{3}$-points $p^{\prime}, p^{\prime \prime}$;

(v) $E$ is the union of four planes having a $R_{4}$-point $p^{\prime}$.

The corresponding dual graphs are illustrated in Figure 4, where the proper transforms of the four components of $X$ concurring at $p$ are the left-hand-side vertices in each graph. As the pictures show, $G$ is a deformation retract of the new dual graph (considered as $\mathrm{CW}$ complexes).

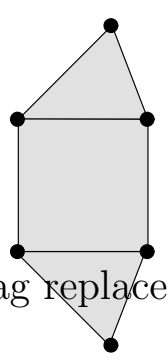

Case (i)

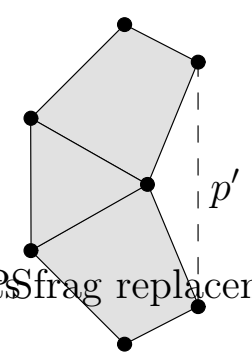

Case (ii)

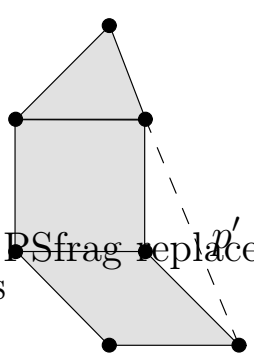

Case (iii)

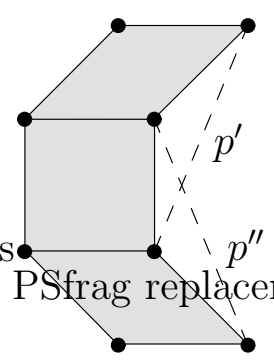

Case (iv)

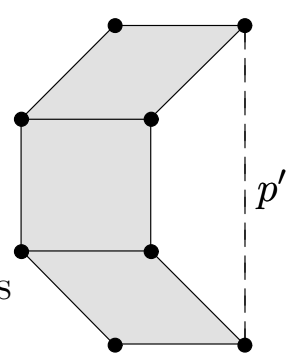

Case (v)

FiguRE 4. After blowing-up a $R_{4}$-point $p$, there are five possibilities

Generally, if one blows-up a $R_{n^{-}}$[resp. $S_{n^{-}}$] point $p$, in the dual graph one builds new 3and 4- faces (triangles and quadrangles) over the original chain of length $n$ [resp. fork with $n-1$ teeth] corresponding to the $n$ components of $X$ concurring at $p$. Therefore it is always the case that $G$ is a deformation retract of the new dual graph.

From this point on there are no more $R_{n}$ or $S_{n}$ points ever appearing in the configuration. However it may happen that at intermediate steps of the algorithm, we do not have strict normal crossings nor Zappatic singularities. If this happens, we still consider the usual dual graph of the configuration, namely a vertex for each component, an edge for each connected component of an intersection between components, and faces for intersections of three or more components.

Consider Step 2 of algorithm 4.8. Each blow-up of a $E_{n}$-point, where the total space has multiplicity $n$, has the effect of adding new vertices in the interior of the corresponding $n$-face and of adding new edges which subdivide the $n$-face. This does not modify the Betti numbers of the dual graph.

In Step 3, the blow-up along a double curve determines a subdivision of the edge corresponding to the double curve and a subdivision of the faces adjacent on that edge.

In Step 4, the blow-ups at double points of types (a) and (b) add trees adjacent only to a vertex or an edge, and again this does not modify the topological properties of the graph. 
Resolving a double point of type (c), one first subdivides the original triangle of vertices $v_{1}, v_{2}, v_{3}$ in three triangles; then, setting $v_{0}$ the new vertex, one adds another vertex $v_{0}^{\prime}$ above $v_{0}$ and three triangles of vertices $v_{0}, v_{0}^{\prime}, v_{i}$, respectively for $i=1,2,3$. Clearly the resulting graph retracts back to a subdivision of the original one.

For a double point of type (d), one subdivides the original quadrangle either in four triangles, if the exceptional divisor $E$ of the blow-up is a smooth quadric, or in two triangles and two quadrangles as in Figure 5 , if $E$ is the union of two planes.

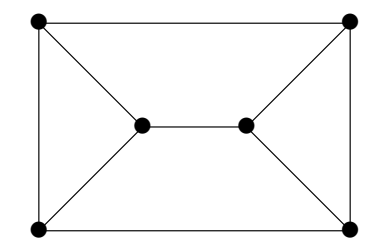

Figure 5. Subdivision of a quadrangle in type (d), case (ii)

For a double point of type (e), one subdivides the original triangle either in three triangles, if the exceptional divisor $E$ of the blow-up is irreducible, or in a triangle and two quadrangles as in Figure 6, if $E$ is reducible.

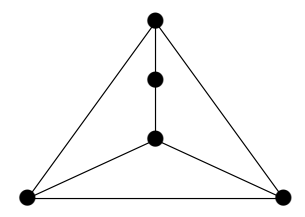

Figure 6. Subdivision of a triangle in type (e), case (iii)

In all cases, one sees that these modifications, coming from the resolution of double points of type (c), (d) and (e), do not change the Betti numbers of the dual graph.

Finally, the blow-ups of Steps 5 and 6 add trees adjacent to a vertex or an edge and again do not modify the Betti number of the dual graph.

We are interested not only in $\bar{X}_{\text {red }}$ but in $\bar{X}$ itself. For each component $i$, let $\mu_{i}$ be the multiplicity of $\bar{X}_{i}$ in $\bar{X}$. For the analysis of the semistable reduction, we must understand rather precisely the components of multiplicity larger than one.

Corollary 4.11. Set $\bar{C}_{i j}=\bar{X}_{i} \cap \bar{X}_{j}$ if $\bar{X}_{i}$ and $\bar{X}_{j}$ meet along a curve, or $\bar{C}_{i j}=\emptyset$ otherwise. If $\mu_{i}>1$, one has the following possibilities:

(i) $\bar{X}_{i}$ is a generically ruled surface and the curve $\sum_{j \neq i} \mu_{j} \bar{C}_{i j}$ is generically supported on a bisection of the ruling.

(ii) There is a birational morphism $\sigma: \bar{X}_{i} \rightarrow \mathbb{P}^{2}$ such that the curve $\sum_{j \neq i} \mu_{j} \bar{C}_{i j}$ maps to four distinct lines.

(iii) $\mu_{i}=4$ and $\bar{X}_{i}$ is a smooth quadric; the curve $\sum_{j \neq i} \mu_{j} \bar{C}_{i j}$ consists of two (multiplicity one) fibres in one ruling and one double fibre from the other ruling.

(iv) $\bar{X}_{i}$ is a smooth quadric and the curve $\sum_{j \neq i} \mu_{j} \bar{C}_{i j}$ is linearly equivalent to $\mu_{i} H$, where $H$ is a plane section of $\bar{X}_{i}$.

(v) There is a birational morphism $\sigma: \bar{X}_{i} \rightarrow \mathbb{P}^{2}$ such that the curve $\sum_{j \neq i} \mu_{j} \bar{C}_{i j}$ is the total transform via $\sigma$ of a plane curve of degree $\mu_{i}$ supported on two distinct lines.

(vi) $\bar{X}_{i}$ is a Hirzebruch surface $\mathbb{F}_{2}$ and the curve $\sum_{j \neq i} \mu_{j} \bar{C}_{i j}$ is of the form $\mu_{i}(H+A)$, where $A$ is the (-2)-curve and $H$ is a section of self-intersection 2. 
Proof. Following the steps of the normal crossing reduction algorithm 4.8, one sees that multiple components are not created in Steps 1 or 2 of the algorithm. It is possible that a multiple component may be created in Step 3, by blowing-up a double curve of $X_{\text {red }}$ which is the intersection of two components that have multiplicity. This will create a multiple ruled surface whose double curve is a bisection, giving case (i).

Multiple components of the central fibre $\bar{X}$ may arise also in Step 4 when one blows-up double points of types (c), (d) and (e). In case (c), two types of multiple components appear. The first is a plane blown-up at three collinear points, with multiplicity two; the double curve consists of the collinearity line, three other general lines, and the three exceptional divisors counted with multiplicity four; this is case (ii). The other type of multiple component is a quadric with multiplicity four, giving case (iii). This analysis follows from the remark we did at the end of Step 4, where we showed that the three surfaces coming together to form this singularity of type (c) each have multiplicity one.

Let $p=X_{1} \cap X_{2} \cap X_{3} \cap X_{4}$ be a point of type (d), where $X_{1}, \ldots, X_{4}$ are irreducible components of $X_{\text {red }}$. One may choose the numbering on the four components such that $X_{1} \cup X_{2}$ and $X_{3} \cup X_{4}$ are local complete intersections of $\mathcal{X}$ at $p$, and moreover the multiplicities satisfy $\mu_{1}=\mu_{2}$ and $\mu_{3}=\mu_{4}$. (This is clear at the start, when all multiplicities are one; and from that point on one proceeds inductively.) Then the exceptional divisor $E$ appears in the new central fibre with multiplicity $\mu_{1}+\mu_{3}=\mu_{2}+\mu_{4}$. Recall that if $E$ is a smooth quadric, the resolution process stops, and we have case (iv) above; while if $E$ is the union of two planes, then both planes appear with multiplicity $\mu_{1}+\mu_{3}$ and we go on inductively; this gives case (v).

Let now $p=X_{1} \cap X_{2} \cap X_{3}$ be a point of type (e). As noted above, $X_{2} \cup X_{3}$ and $X_{1}$ are local complete intersections of $X$ at $p$. As above, one may assume that the multiplicities satisfy $\mu_{2}=\mu_{3}$. Then the exceptional divisor $E$ appears in the new central fibre with multiplicity $\mu_{1}+\mu_{2}=\mu_{1}+\mu_{3}$. If $E$ is a smooth quadric, the resolution process stops, giving case (iv) again. If $E$ is a quadric cone, then we proceed to blow-up the vertex of the cone, and therefore the proper transform of $E$ in the final central fibre will be a Hirzebruch surface $\mathbb{F}_{2}$, which gives the final case (vi). Finally if $E$ is a pair of planes, each plane gives rise to a component in case $(\mathrm{v})$.

Now we are able to prove the main result of this section:

Proposition 4.12. Let $\pi: \mathcal{X} \rightarrow \Delta$ be a degeneration of surfaces with Zappatic central fibre $X=X_{0}=\bigcup_{i=1}^{v} X_{i}$. Let $\bar{\pi}: \bar{X} \rightarrow \Delta$ be the normal crossing reduction of $\pi$ given by algorithm 4.8 and let $\tilde{\pi}: \tilde{X} \rightarrow \Delta$ be the semistable reduction of $\bar{\pi}$ obtained by following the process described in Chapter II of [9]. Then:

$$
p_{\omega}\left(\tilde{X}_{0}\right)=p_{\omega}(X) .
$$

Proof. Let $\bar{X}=\bar{X}_{0}=\sum_{i=1}^{w} \mu_{i} \bar{X}_{i}$ be the central fibre of the normal crossing reduction $\bar{\pi}$. One has $v \leqslant w$ and we may assume that $\mu_{i}=1$ for $1 \leqslant i \leqslant v$, and that these first $v$ components are birational to the original components of $X$. The surface $\bar{X}$ is a toroidal embedding in $\bar{X}$, in the sense of Definition 1, p. 54 of [9]. To any such a toroidal embedding one can associate a compact polyhedral complex $\bar{\Gamma}$ with integral structure as shown in [9], pp. 71 and 94 . In our present situation, the complex $\bar{\Gamma}$ is exactly the dual graph $\bar{G}$. The integral structure is recorded by the multiplicities of the components.

By [9], p. 107, there exists a semistable reduction $\tilde{X} \rightarrow \Delta$ as in Diagram 4.3 , where the base change $\beta(t)=t^{m}$ is such that $m$ is a common multiple of $\mu_{1}, \ldots, \mu_{w}$. Notice that $\tilde{X}$ is again a toroidal embedding of the central fibre $\tilde{X}=\tilde{X}_{0}$. Denote by $\tilde{G}$ the dual graph of $\tilde{X}$. Again by [9], p. 107, one has that the corresponding polyhedron $\tilde{\Gamma}$ is a subdivision of $\bar{\Gamma}$, in 
the sense of the definition at p. 111 of [9]. This implies that the CW-complexes $\tilde{G}$ and $\bar{G}$ are homeomorphic. In particular they have the same homology.

Now the central fibre $\tilde{X}=\tilde{X}_{0}=\bigcup_{i=1}^{u} \tilde{X}_{i}$ is reduced, with global normal crossings. One has that $u \geqslant w$ and, by taking into account the base change, one may assume that, for $i=1, \ldots, w, \tilde{X}_{i}$ is birational to the $\mu_{i}$-tuple cover of $\bar{X}_{i}$, branched along $\sum_{j \neq i} \mu_{j} \bar{C}_{i j}$.

Let us first consider components with $\mu_{i}=1$. These include the first $v$ components $\tilde{X}_{i}$, $i=1, \ldots, v$, which correspond to the original components of $X$. For these components we have $p_{g}\left(\tilde{X}_{i}\right)=p_{g}\left(\bar{X}_{i}\right)=p_{g}\left(X_{i}\right), i=1, \ldots, v$. There also may be components with $\mu_{i}=1$ which were introduced in the normal crossing reduction algorithm. We have seen in Proposition 4.10 that all such components have $p_{g}=0$. Finally there may be components with $\mu_{i}=1$ with $i>w$ which have been introduced in the semistable reduction process. These new surfaces are of two types: they may correspond either to

(a) vertices of $\tilde{G}$ which lie on an edge $\eta$ of $\bar{G}$; or to

(b) vertices of $\tilde{G}$ which lie in the interior of a triangular face of $\bar{G}$.

We recall that the birational morphism $\tilde{X} \rightarrow X_{\beta}$ as in Diagram 4.3 is the blow-up of a suitable sheaf of ideals, cf. p. 107 of [9].

Let $\tilde{X}_{j}$ be a surface of type (a). This is an exceptional divisor of such a blow-up with support on the double curve $\gamma$ of $\bar{X}$ corresponding to the edge $\eta$. Then $\tilde{X}_{j}$ maps to $\gamma$ with fibres which are rational by the toric nature of the singularity along $\gamma$.

Suppose that $\tilde{X}_{j}$ is of type (b). Then $\tilde{X}_{j}$ is an exceptional divisor appearing in the toric resolution of a toric singular point. Therefore $\tilde{X}_{j}$ is rational and moreover it meets the other components along rational curves (cf., e.g., Section 2.6 in [7]).

Therefore all of these components are rational or ruled, and hence also have $p_{g}=0$.

Now let us consider the case $\mu_{i}>1$. In this case $\tilde{X}_{i}$ is a $\mu_{i}$-cover of the surface $\bar{X}_{i}$, and such surfaces were classified in the previous corollary, along with the double curves which give the branch locus of the covering. In each case the cover is easily seen to be rational or ruled. Hence also for these surfaces one has $p_{g}=0$.

Since we have shown that the homology of the graphs are the same, and we have controlled the $p_{g}$ of the components properly, the only thing left to prove is that $\operatorname{dim}\left(\operatorname{coker}\left(\Phi_{X}\right)\right)=$ $\operatorname{dim}\left(\operatorname{coker}\left(\Phi_{\tilde{X}}\right)\right)$.

We have already seen that $\operatorname{dim}\left(\operatorname{coker}\left(\Phi_{\bar{X}_{\text {red }}}\right)\right)=\operatorname{dim}\left(\operatorname{coker}\left(\Phi_{X}\right)\right)$ in Proposition 4.10. The argument here is similar; it suffices to show that the extra components $\tilde{X}_{v+1}, \ldots, \tilde{X}_{u}$ do not contribute to $\operatorname{dim}\left(\operatorname{coker}\left(\Phi_{\tilde{X}}\right)\right)$. These surfaces are either rational or ruled over a curve $\gamma$. In the rational case, by the proof of Proposition 4.8 and by the above considerations about toric resolution of singularities, they meet the other components of $\tilde{X}$ along rational curves. Hence they do not contribute to $\operatorname{dim}\left(\operatorname{coker} \Phi_{\tilde{X}}\right)$.

In the ruled case, $\tilde{X}_{j}$ is a scroll over $\gamma$ and, by the description of the resolution process, $\tilde{X}_{j}$ meets the other components of $\tilde{X}$ along curves which are either rational or isomorphic to $\gamma$. The same argument as in Proposition 4.10 shows that the cokernel is unchanged in this case.

Thus the proof is concluded by Theorem 3.1.

As a direct consequence, we have the following:

Theorem 4.14. Let $\pi: X \rightarrow \Delta$ be a degeneration of surfaces with Zappatic central fibre $X=X_{0}$. Then, for any $t \neq 0$, one has:

$$
p_{g}\left(X_{t}\right)=p_{\omega}(X) .
$$


Proof. Just consider the semistable reduction $\tilde{\pi}: \tilde{X} \rightarrow \Delta$ as we did before. One clearly has that $p_{g}\left(\mathcal{X}_{t}\right)=p_{g}\left(\tilde{X}_{t}\right)$ for $t \neq 0$. Theorem 4.4 then implies that $p_{g}\left(\tilde{X}_{t}\right)=p_{\omega}\left(\tilde{X}_{0}\right)$ and finally Proposition 4.12 concludes that $p_{\omega}\left(\tilde{\mathcal{X}}_{0}\right)=p_{\omega}(X)$.

\section{REFERENCES}

[1] Calabri, A., Ciliberto C., Flamini, F., Miranda, R., On the geometric genus of reducible surfaces and degenerations of surfaces to union of planes, Proceedings of the Fano Conference - Torino (I), 29 September - 5 October 2002 (2004), 277 - 312.

[2] Calabri, A., Ciliberto C., Flamini, F., Miranda, R., On the $K^{2}$ of degenerations of surfaces and the multiple point formula, to appear on Annals of Mathematics.

[3] Ciliberto, C., Lopez, A. F., Miranda, R., Projective degenerations of $K 3$ surfaces, Gaussian maps and Fano threefolds, Inv. Math., 114 (1993), 641-667.

[4] Ciliberto, C., Miranda, R., Teicher, M., Pillow degenerations of K3 surfaces, in Application of Algebraic Geometry to Computation, Physics and Coding Theory, Nato Science Series II/36, Ciliberto et al. (eds.), Kluwer Academic Publishers, 2002.

[5] Cohen, D. C., Suciu, A. I., The braid monodromy of plane algebraic curves and hyperplane arrangements, Comment. Math. Helv., 72 (1997), 285-315.

[6] Friedman, R., Morrison, D.R., (eds.,) The birational geometry of degenerations, Progress in Mathematics 29, Birkhauser, Boston, 1982.

[7] Fulton, W., Introduction to toric varieties, Annals of mathematics studies 131, Princeton University Press, 1993.

[8] Hartshorne, R., Families of curves in $\mathbb{P}^{3}$ and Zeuthen's problem, Mem. Amer. Math. Soc. 130 (1997), no. 617.

[9] Kempf, G., Knudsen, F.F., Mumford, D., and Saint-Donat, B., Toroidal embeddings. I., Lecture Notes in Mathematics 339, Springer-Verlag, Berlin-New York, 1973.

[10] Kollár, J., Toward moduli of singular varieties, Compositio Mathematica, 56 (1985), 369-398.

[11] Moishezon, B., Stable branch curves and braid monodromies, in Algebraic geometry (Chicago, Ill., 1980), Lecture Notes in Math., 862 (1981), Springer , 107-192.

[12] Moishezon, B., Teicher, M., Braid group techniques in complex geometry III: Projective degeneration of $V_{3}$, in Classification of Algebraic Varieties, Contemporary Mathematics 162, AMS (1994), 313-332.

[13] Morrison, D.R., The Clemens-Schmid exact sequence and applications, in Topics in Trascendental Algebraic Geometry, Ann. of Math. Studies, 106 (1984), 101-119.

[14] Severi, F., Vorlesungen über algebraische Geometrie, vol. 1, Teubner, Leipzig, 1921.

[15] Teicher, M., Hirzebruch surfaces: degenerations, related braid monodromy, Galois covers. in Algebraic geometry: Hirzebruch 70 (Warsaw, 1998), Contemp. Math., 241 (1999), 305-325.

[16] Zappa, G., Su alcuni contributi alla conoscenza della struttura topologica delle superficie algebriche, dati dal metodo dello spezzamento in sistemi di piani, Acta Pont. Accad. Sci., 7 (1943), 4-8.

[17] Zappa, G., Alla ricerca di nuovi significati topologici dei generi geometrico ed aritmetico di una superficie algebrica, Ann. Mat. Pura Appl., 30 (4) (1949), 123-146.

E-mail address: calabri@dm.unibo.it

Current address: Dipartimento di Matematica, Università degli Studi di Bologna, Piazza di Porta San Donato, 5 - 40126 Bologna, Italy

E-mail address: cilibert@mat.uniroma2.it

Current address: Dipartimento di Matematica, Università degli Studi di Roma "Tor Vergata", Via della Ricerca Scientifica, 00133 Roma, Italy

E-mail address: flamini@mat.uniroma3.it

Current address: Dipartimento di Matematica Pura ed Applicata, Università degli Studi di L'Aquila, Via Vetoio, Loc. Coppito, 67100 L'Aquila, Italy

E-mail address: miranda@math.colostate.edu

Current address: Department of Mathematics, 101 Weber Building, Colorado State University, Fort Collins, CO 80523-1874, U.S.A. 